\title{
Expert system for Robotic Path Planning
}

\author{
Baidaa M. Madlol, Ahmad T. Abdulsadda, Ali A. Al Bakry
}

\begin{abstract}
Robotic planning to find the target our goal point/s is most important subject with the minimum distance and the fastest speed with obstacle avoidance expert system has been proposed. In this paper we try to compare and consider different scenario by taking two or more moving robot figure out the short path from the initial and the final point automatically through the map of many regular and irregular obstacles. Firstly, the adaptive fuzzy expert system is present where the fuzzy rule has been adaptive recursively through the robot moving, and then the potential field algorithm has been compared with the adaptive fuzzy system, the results demonstrated that the adaptive fuzzy is faster than the potential field but the accuracy moving of the potential field robotic path planning is much better. All the algorithms were failed when two robots moving from two different initial points to one final target point the why we have proposed particle swarm optimization (PSO) algorithm to solve such problem.
\end{abstract}

Keywords : robot, path planning, optimization, fuzzy, PSO.

\section{INTRODUCTION}

At present portable robots have been effectively utilized in different engineering of designing for example, aviation examine, atomic research, generation designing and so forth. The significant target in the flow automated research field is to discover an impact freeway from a given beginning situation to predefined goal point. When all is said in done way arranging calculations are delegated neighborhood and worldwide relying on the encompassing condition. In worldwide way arranging the encompassing condition is totally known to the portable robot so the way went by the versatile robot is predefined, whereas in neighborhood way arranging the earth is totally obscure or halfway known to the versatile robot so different sensors are used to see the data about the encompassing condition and plan the movement in like manner. Numerous efforts have been paid in the past to improve different robot route calculations.

In state of the art, there can be discovered a few scientists have been tended to on numerous canny strategies for portable robot route. Numerous creators have thought about a

Revised Manuscript Received on February 05, 2020.

* Correspondence Author

Baidaa M. Madlol*, Communication Technical Engineering Department ,Al Najaf Technical Engineering College, Al Furat Al Awast Technical University (ATU), Al Najaf(54001), Iraq,. Email:baidaa.alkhlidi@yahoo.com.

Ahmad T. Abdulsadda, Communication Technical Engineering Department , Al Najaf Technical Engineering College, Al Furat Al Awast Technical University (ATU), Al Najaf(54001), Iraq,. Email: coj.abdulsad@atu.edu.iq.

Ali A. Abdullah Albakry, Communication Technical Engineering Department ,Al Najaf Technical Engineering College, Al Furat Al Awast Technical University (ATU), Al Najaf, Iraq,. Email:aaali@yahoo.com.

(C) The Authors. Published by Blue Eyes Intelligence Engineering and Sciences Publication (BEIESP). This is an open access article under the CC BY-NC-ND license (http://creativecommons.org/licenses/by-nc-nd/4.0/) controller with complete data of the earth [1-2].Due to the unpredictability and vulnerability of the way arranging issue, old style way arranging strategies, for example, Visibility Graph [3], Voronoi outlines [4], Matrices [5], Cell disintegration [6], counterfeit potential field [7], Rule based strategies [8], and Rules learning methods [9] are not fitting for way arranging in powerful conditions. The utilization of the above calculations for way finding for portable robot requires additional time and the finding of this way won't totally doable for constant development. There are numerous fluffy rationale techniques utilizing different executions or in blend with different systems [10-14]. In worldwide way arranging issue the earth will be acquainted with the robot while in nearby way arranging the robot is oblivious of the encompassing. For taking care of the advancement issue a socially roused famous meta-heuristic improvement calculation is utilized; which is known as Particle Swarm Optimization (PSO) method. PSO was motivated by the social conduct of feathered creatures and fish. Molecule Swarm Optimization (PSO) was created by Eberhart and Kennedy [15-20], propelled by social conduct of winged animal running or fish tutoring. Numerous past specialists have been utilized this calculation to comprehend way anticipating a portable robot. In [18, 21], creators have thought about a lot of versatile robots as swarm and they actualized the multi-robot-multi-target search with same wellness work in the work space. This article manages age of ideal impact free directions of different portable robots from their beginning to goal position with both static and dynamic obstructions. To achieve this ideal errand another wellness work is proposed in this paper which considers parameters like separation between obstruction - robot, robot-objective and robot-robot. Reenactment results are given to check the effectiveness of the created calculation. The rest of the paper is organized as: section II presents a mathematical model for the adaptive fuzzy, potential filed, and finally the adaptive PSO algorithms, respectively. Section III presents the simulation setup and the algorithms flowcharts. The simulation rsults would be discussed in section IV, finally the discussion and future works would be briefly discussed in section $\mathrm{V}$.

\section{MATHEMATICAL FORMULATION}

\section{A. Adaptive fuzzy expert system}

The perusers ought to please acquaint themselves with Fuzzy Logic before perusing this instructional exercise. Fluffy based route is a responsive arranging method, where the quick position and good ways from impediments is considered to figure the prompt move, absent really any making a big deal about what's to come. 
In such a way prompt activities lead to movement of the robot, at last prompting the objective. So as to tackle the issue utilizing fluffy rationale, we first need to choose a couple of information sources which best speak to the circumstance that the robot is right now put in. The choice of movement is made simply based on these sources of info and not the genuine situation. For this issue 6 data sources are chosen. These are good ways from the snag in front, good ways from the deterrent at the front left inclining, good ways from the obstruction at the front right corner to corner, point between the heading course of robot and the objective, separation of the robot from the objective and favored turn. The various information sources are condensed in Figure 1.

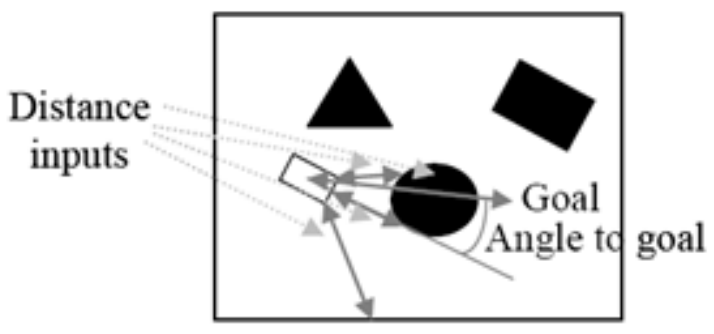

Fig.1: Adaptive fuzzy rules robotic path planning, adaptive, [3].

The last info, favored turn demonstrates whether it is helpful to turn clockwise or hostile to clockwise, all different sources of info disregarded. A straightforward guideline is utilized to set the parameter. In the event that the front impediment is far away, turn is in order to more face the objective. In the event that the front impediment is close and another front hindrance is experienced, turn utilizing the side of the objective is liked. On the off chance that the front hindrance is close and a similar impediment as experienced in the past advance is discovered, a similar turn as made beforehand is rehashed.

The fluffy framework delivers a solitary yield, which is the controlling to make or the quick precise speed. The fluffy principles are composed to such an extent that the robot keeps away from the impediments and adjusts itself towards the objective. The fluffy framework is an aftereffect of a great deal of manual tuning of the guidelines and participation capacities, over a wide assortment of situations. Figure 2 is shown the an example of the three fuzzy rule for the one front obstacle map.

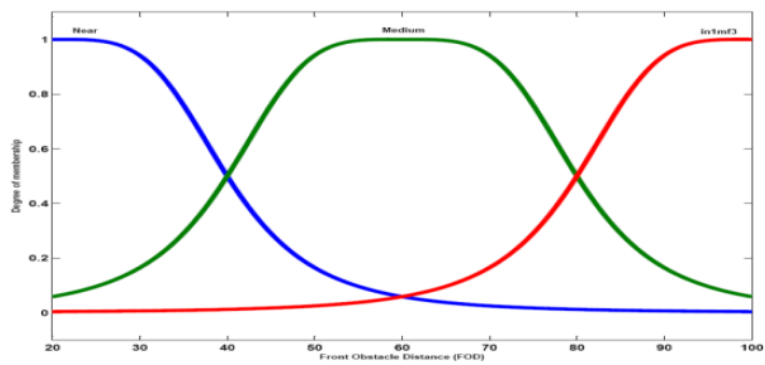

Fig. 2: Three fuzzy front obstacle.

\section{B. Potential field expert system}

The reader ought to please acquaint themselves with Artificial Potential Fields before perusing this instructional exercise. Fake Potential Field based route is a responsive arranging procedure, where the prompt good ways from impediments are considered to register the quick move, absent really any fretting over what's to come. In such a way quick activities lead to movement of the robot, at last prompting the objective. All hindrances repulse the robot with a greatness conversely relative to the separation. The objective draws in the robot. The resultant potential, representing the appealing and terrible segments is estimated and used to move the robot. The potential field for an example situation is appeared in Figure 2. Bearings demonstrate the heading of the potential vector.

The separation of the obstructions at all edges from the robot is estimated. In this instructional exercise just 5 separations at explicit points are estimated to figure the horrendous potential. These are forward, left side, right side, forward left slanting and forward right corner to corner. The various data sources are outlined in Figure 3.

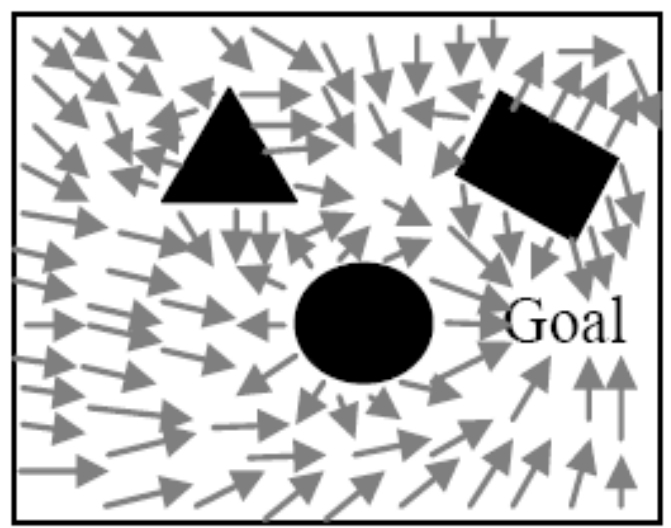

C. Adaptive particle swarm optimization expert system

As referenced before, Particle Swarm Optimization procedure is enlivened by the social conduct of feathered creatures and fishes. On account of fledgling running, when winged creatures move in bunch then each fowl in the herd is having its own position and speed. This is only the individual best situation of each winged creature. The best situation in the herd is measured dependent on an information parameter known as target capacity or wellness work. If there should arise an occurrence of feathered creature running, the wellness work parameter is scanning for nourishment in less good ways from the haven of winged animals. So each feathered creature is having some close to home best position dependent on this wellness work. The situation of the winged creature which has ideal wellness esteem is considered as the worldwide best position. As the winged animals share data among them, all fowls will in general move towards the worldwide best position. As PSO is propelled by the social idea of winged creature running, each fledgling can be considered as one molecule.

So every molecule has its very own position and speed. The situation of molecule which fulfill ideal wellness esteem measure is picked as the worldwide best position. Figure.4 beneath shows the structure of PSO.

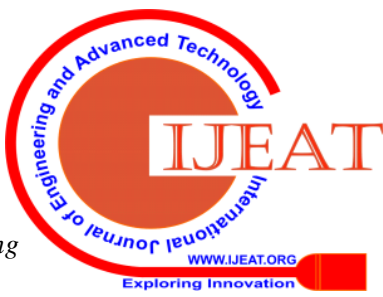




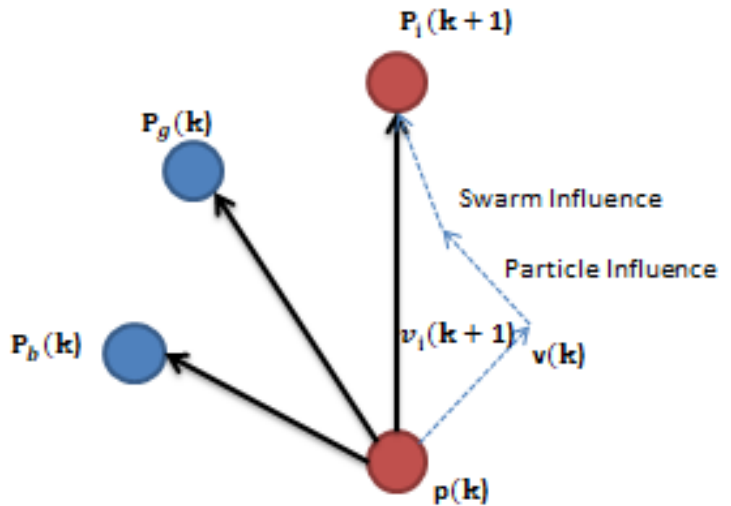

Fig. 4: PSO path planning,[18].

Consider at first, the robot $\mathrm{Ri}$ is set in the area at ( $\mathrm{xi}^{\text {curr }}$ , $\left.\mathrm{yi}^{\mathrm{curr}}\right)$. We need to locate the following area of the robot ( $\left.\mathrm{xi}^{\text {next }}, \mathrm{yi}^{\text {next }}\right)$ by joining of the two focuses between $\left\{\left(\mathrm{xi}^{\text {curr }}\right.\right.$, $\left.\left.\mathrm{yi}^{\text {curr }}\right),\left(\mathrm{xi}^{\text {next }}, \mathrm{yi}^{\text {next }}\right)\right\}$ and $\left\{\left(\mathrm{xi}^{\text {next }}, \mathrm{yi}^{\text {next }}\right) ;\left(\mathrm{xi}^{\text {goal }}, \mathrm{yi}^{\text {goal }}\right)\right\}$ ought not contact the deterrent on the planet map, as spoke to in Figure 5, and limits the all out way length from current situation to an objective situation without contacting the obstruction by framing limitation. At that point the target work F1, which decides the direction way length for $n$, number of robots, is

$$
F_{1}=\sum_{i=1}^{n}\left\{\sqrt{\left(\left(x_{i}^{\text {curr }}-x_{i}^{\text {next }}\right)^{2}+\left(y_{i}^{\text {curr }}-y_{i}^{\text {next }}\right)^{2}\right)}\right.
$$

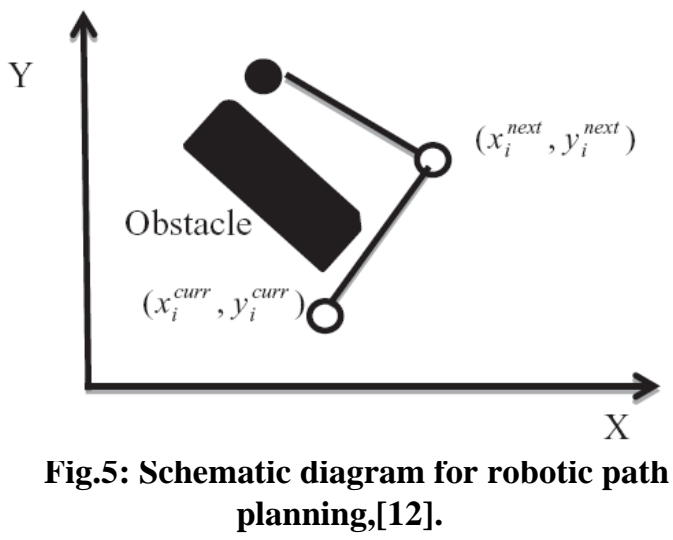

\section{SIMULATION SETUP AND ALGORITHMS}

The main steps for the all expert systems can be defined by the following procedure :

1. Characterize robot and objective position. Characterize greatest number of cycles.

2. Move the robot from its source to goal by augmenting the estimation of level and vertical segment of the position vector.

3. In the event that there is no hindrance the robot will move to goal toward incline among source and goal since slant is the most limited way between any two focuses.

4. On the off chance that the robot detects any obstruction before it creates irregular particles and dependent on least wellness esteem criteria the worldwide best position is found.

5 . The robot will move to these worldwide best situations until it arrives at the goal. At the point when it will arrive at the goal the emphasis stops.

Firstly we have to define the mapping for the robot, this can be done by using the paint program to plot the obstacles and read the map directly from the robot sensors, or it can be generated by the matlab platform directly. Figure 6 is shown one example for the path robotic planning generated by the paint program.
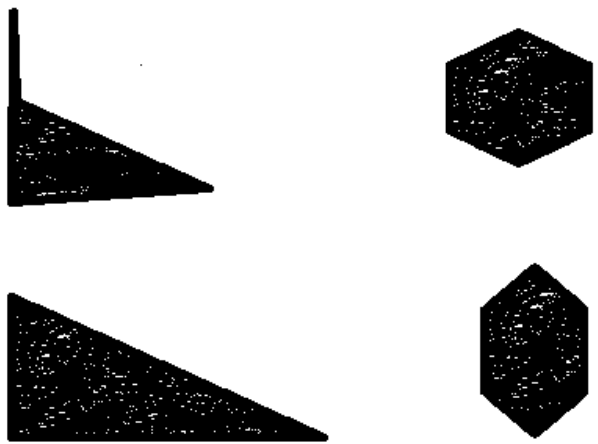

Fig.

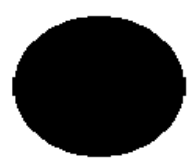

\section{SIMULATION RESULTS}

\section{A. Adaptive fuzzy path planning}

So as to execute the code you have to execute the record 'fuzzy.m'. First make another bitmap record and draw any guide on it, over which you have to execute the calculation. Paint or some other straightforward drawing instrument can be utilized. Ensure you spare the document as a BMP. Spot the record in the undertaking envelope. You may want to open any of the provided maps and re-draw them. Change the name of the guide document in the code to call attention to the guide that you made. Supply the source and the objective positions and the underlying heading course (in radians). You can utilize paint or some other drawing utility which shows the pixel places of the focuses, to find the source and objective on your drawn guide. Robot static and kinematic parameters might be changed to suit the necessities. Anyway this will change the perfect fluffy surmising framework. Changes to the principles and the enrollment capacities can be conveyed at the fluffy editorial manager.

Execute the calculation. You should take screen captures of the presentation, the instrument doesn't do that for you. The reenactment will stop when the robot arrives at its objective, impacts or is compelled to stop according to the wellbeing safeguards. The way length and execution time are given at the support. Figure 7 shown the final path find for the adaptive fuzzy expert system. 


\section{Expert system for Robotic Path Planning}

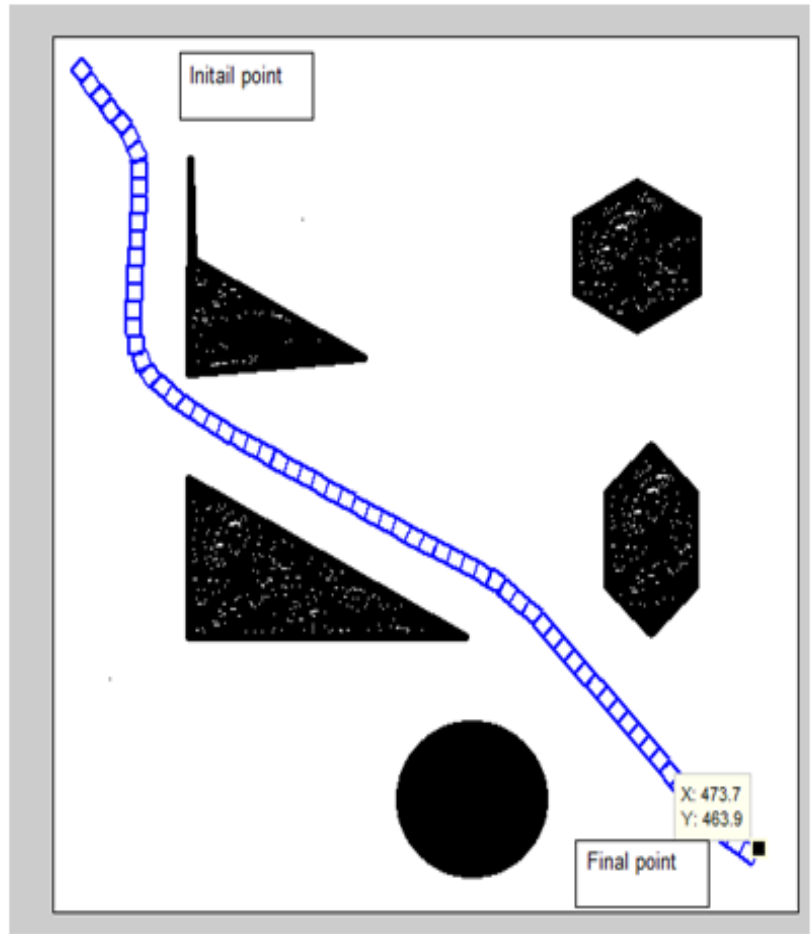

Fig. 7: robotic moving through the obstacles using adaptive fuzzy expert system.

\section{B. Potential field algorithm}

So as to execute the code you have to execute the record 'potential.m'. First make another bitmap record and draw any guide on it, over which you have to execute the calculation. Paint or some other straightforward drawing instrument can be utilized. Ensure you spare the document as a BMP. Spot the record in the undertaking envelope. You may want to open any of the provided maps and re-draw them. Change the name of the guide document in the code to call attention to the guide that you made. Supply the source and the objective positions and the underlying heading course (in radians). You can utilize paint or some other drawing utility which shows the pixel places of the focuses, to find the source and objective on your drawn guide. Robot static and kinematic parameters might be changed to suit the necessities. Anyway this will change the perfect fluffy surmising framework. Changes to the principles and the enrollment capacities can be conveyed at the fluffy editorial manager. Execute the calculation. You should take screen captures of the presentation, the instrument doesn't do that for you. The reenactment will stop when the robot arrives at its objective, impacts or is compelled to stop according to the wellbeing safeguards. Figure 8 shown the fail reach robotic moving using the potential field algorithm.

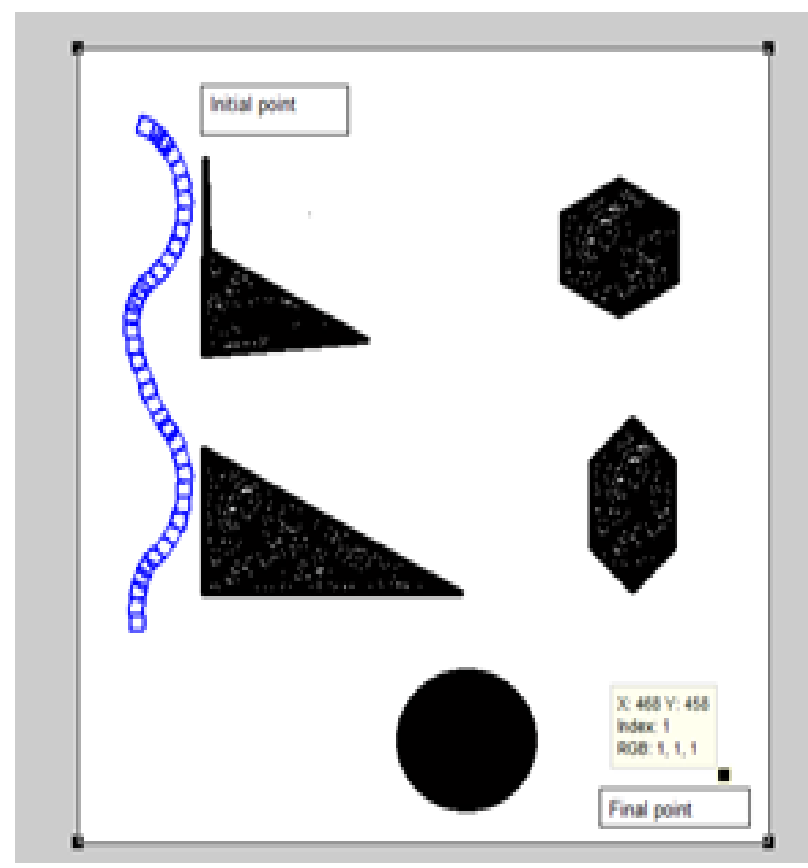

Fig.8: Potential field doesn't reach the goal point.

\section{PSO algorithm}

The recreation of robot way direction is plotted utilizing MATLAB 2013b. For producing diverse impact free directions the tuning parameter $\mathrm{z}$ is tuned appropriately. The recreation is performed by picking $c_{1}=1$ and $c_{2}=1$. However various qualities for $\mathrm{c} 1$ and $\mathrm{c} 2$ can be picked. The estimation of $\mathrm{r} 1$ and $\mathrm{r} 2$ are picked as one for effortlessness. In the wake of tuning the parameter, it is discovered that the parameter at $z=0.36$ gives better outcomes. The reproduction result approving the above articulations demonstrated as follows as shown in figure 9:

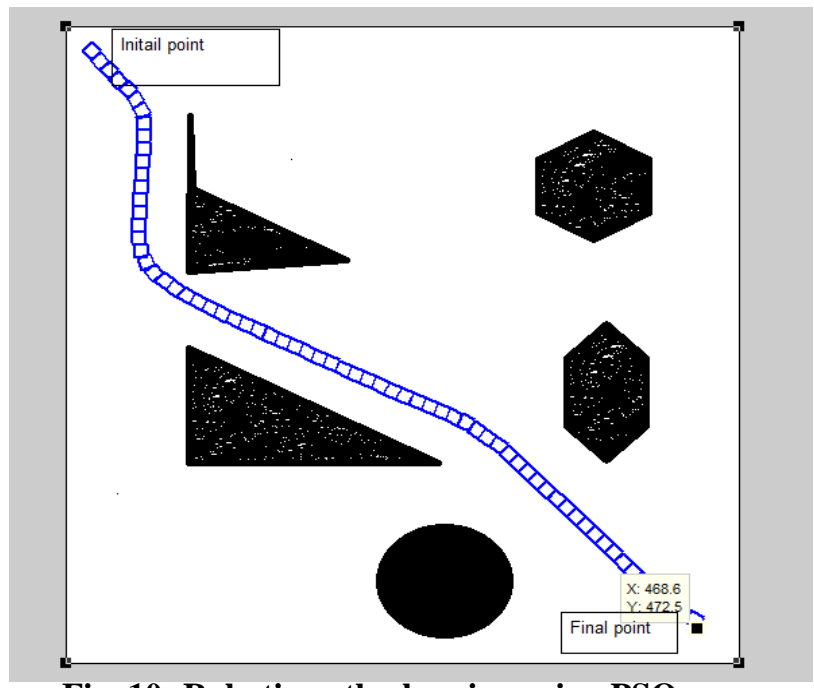

Fig. 10: Robotic path planning using PSO.

Generally, from the above results the adaptive fuzzy and the PSO is reach the target point through the obstacles, while the potential algorithms hit the obstacle and stop the program. Table I listed the numerical results of the three schemes.

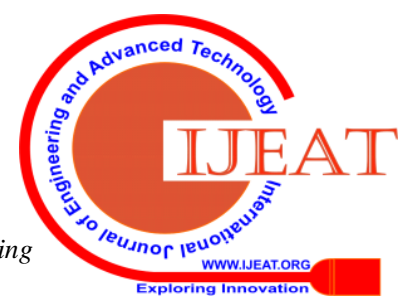


Table- I: Performance time and accuracy.

\begin{tabular}{|l|c|c|c|}
\hline \multirow{2}{*}{ No. } & \multicolumn{3}{|c|}{ Table Column Head } \\
\cline { 2 - 4 } & Adaptive fuzzy & Potential & PSO \\
\hline Path length & 904 & 300 & 904 \\
\hline $\begin{array}{c}\text { Execution } \\
\text { Time (sec.) }\end{array}$ & 1.08 & 2 & 1.16 \\
\hline
\end{tabular}

\section{CONCLUSION AND FUTURE WORKS}

In this paper way arranging of portable robots have been tended to. The created movement organizer works for the automated condition with static and moving deterrents. So as to maintain a strategic distance from the detected obstructions (static and dynamic) and create briefest way, another wellness work has been presented. To approve the effectiveness of the proposed approach, reenactment results have been displayed in the different automated conditions. The adaptive fuzzy and the adaptive PSO can be detected and avoid the hard obstacles while the potential failed when the map has difficult irregular obstacles.

In future the created calculation is to be executed in automated stage. Build a hard ware to demonstrate the simulation results, taken two moving synchronize robots.

\section{REFERENCES}

1. Latombe J. C., Robot motion planning, 124, Springer Science and Business Media, 2012.

2. Brooks R. A., A robust layered control system for a mobile robot, IEEE Journal of Robotics and Automation, 2(1), 1986, 14-23.

3. Mitchell Joseph.S.B., An algorithm approach to some problems in terrain navigation, Artificial Intelligence, 37(1-3), 1988, 171-201.

4. Masehian E. and Sedighizadeh D., Classic and heuristic approaches in robot motion planning-a chronological review, World Academy of Science, Engineering and Technology, 23, 2007, 101-106.

5. Stoeter S.A. and Papanikolopoulos N., Kinematic motion model for jumping scout robots, IEEE Transactions on Robotics, 22(2), 2006, 398-403.

6. Hosoda K., Takuma T., Nakamoto A. and Hayashi S., Biped robot design powered by antagonistic pneumatic actuators for multi-modal locomotion, Robotics and Autonomous systems, 56(1), 2008, 46-53.

7. Wu X. and Ma S., CPG-based control of serpentine locomotion of a snake-like robot, Mechatronics, 20(2), 2010, 326-334.

8. Bayraktaroglu Z.Y., Snake-like locomotion: Experimentations with a biologically inspired wheel-less snake robot, Mechanism and Machine theory, 44(3), 2009, 591-602.

9. Guzman J.L., Berenguel M., Rodriguez F. and Dormido S., An interactive tool for mobile robot motion planning, Robotics and Autonomous systems, 56(5), 2008, 396-409.

10. Alexander J.C. and Maddocks J.H., On the kinematics and control of wheeled mobile robots, SRC TR 87-196, October 1987, 1-15.

11.Campion G., Bastin G., and AndrCa-Novel B.D., Structural properties and classification of kinematic and dynamic models of wheeled mobile robots, IEEE Transactions on Robotics and Automation, 12(1), 1996, 47-62.

12. Kim D.S., Kwon W.H. and Park H.S., Geometric kinematics and applications of a mobile robot, International Journal of Control, Automation, and Systems,1(3), 2003, 376-384.

13. Cariou C., Lenain R., Thuilot B. and Martinet P., Adaptive control of four-wheel-steering off-road mobile robots: Application to path tracking and heading control in presence of sliding, IEEE International conference on Intelligent Robots and Systems, France, September 22-26, 2008, 1759-1764.

14.Marcovitz F.R. and Kelly A., On-line mobile robot model identification using integrated perturbative dynamics, Experimental Robotics, Springer Berlin Heidelberg, January, 2014, 417-431.

15.Lee J.H., Kim B.K., Tanikawa T. and Ohba K., Kinematic analysis on omni-directional mobile robot with double-wheel-type active casters, IEEE International conference on Control, Automation and Systems, Seoul, Korea, October 17-20, 2007,1217-1221.
16. Chung W., Moon C., Jung C. and Jin J., Design of the dual offset active caster wheel for holonomic omni-directional mobile robots, International Journal of Advanced Robotic Systems, 7(4), 2010, 105-110.

17.Li Y.P., Zielinska T., Ang Jr. M.H. and Lin W., Vehicle Dynamics of Redundant Mobile Robots with Powered Caster Wheels, Springer Vienna, 221-228.

18. Kim S. and Lee S., Local and global isotropy analysis of mobile robots with three active caster wheels, Advances in service robotics, ISBN 978-953-7619-02-2, 2008,117-126.

19. Indiveri G., Swedish wheeled omnidirectional mobile robots: kinematics analysis and control, IEEE Transactions on Robotics, 25(1), 2009, 164-171.

20. Indiveri G., Paulus J. and Ploger P.G., Motion control of swedish wheeled mobile robots in the presence of actuator saturation, LNCS 4434, Springer Berlin Heidelberg, 2007, 35-46.

21. Doroftei I., Grosu V. and Spinu V., Omnidirectional mobile robot-design and implementation, Bio-inspiration and robotics: walking and climbing robots, ISBN 978-3-902613-15-8, In-Tech publishers, 2007, 511-528.

\section{AUTHORS PROFILE}

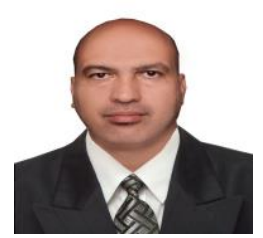

Baidaa M. Madlol: received the B. Sc. degree in communication technical engineering from Al Furat Al Awast Technical University, Iraq in 2006. Currently he is a candidate to pursue a M. Sc. degree in communication technical engineering from Al Furat Al Awast Technical university since 2018.

Dr.Ahmad T. Abdulsadda received the B. Sc. degree in electrica engineering from Tickrit University, Iraq in 1997, M. Sc. degree in electrical engineering from Baghdad University, Iraq in 2000, and the PHd in Electrical and Computer Engineering Department at Michigan State University, Michigan, USA. . From 2000 to 2006, he was a faculty member at Baghdad University, Iraq. Since 2006, he has been a faculty member at Technical Najaf College, Al Furat Al Awast University, Iraq. Currently, he is an assistance dean of Al Najaf Technical engineering college, Al Furat Al Awast Technical University. His research interests include robotics fish, feedback control systems, nonlinear estimation techniques, and control theory.

Prof. Dr. Ali A. Abdullah Albakry: received the B. Sc. degree in electrical engineering from Tickrit University, Iraq in 1980, M. Sc. degree in electrical engineering from Al Technology University, Iraq in 2000, and the PHd in Electrical and Computer Engineering Department at , $\mathrm{Al}$ Technology University, 2008, Iraq. 